\title{
Development of a Natural Language Processing Tool to Extract Radiation Treatment Sites
}

Gary Walker ${ }^{1}$, Ergin Soysal ${ }^{2}$, Hua Xu ${ }^{2}$

1. Radiation Oncology, Banner MD Anderson Cancer Center, Gilbert, USA 2. School of Biomedical Informatics, The University of Texas Medical School at Houston, Houston, USA

Corresponding author: Gary Walker, gary.walker@bannerhealth.com

\begin{abstract}
Currently, radiation oncology-specific electronic medical records (EMRs) allow providers to input the radiation treatment site using free text. The purpose of this study is to develop a natural language processing (NLP) tool to extract encoded data from radiation treatment sites in an EMR.
\end{abstract}

Treatment sites were extracted from all patients who completed treatment in our department from April 1, 2011, to April 30, 2013. A system was designed to extract the Unified Medical Language System (UMLS) concept codes using a sample of 11,018 unique site names from 31118 radiation therapy (RT) sites. Among those, 5500 unique site name strings that constitute approximately half of the sample were spared as a test set to evaluate the final system. A dictionary and calculated n-gram statistics using UMLS concepts from related semantic types were combined with manually encoded data.

There was an average of 2.2 sites per patient. Prior to extraction, the 20 most common unique treatment sites were used 4215 times (38.3\%). The most common treatment site was whole brain RT, which was entered using 27 distinct terms for a total of 1063 times. The customized NLP solution displayed great gains as compared to other systems, with a recall of 0.99 and a precision of 0.99 .

A customized NLP tool was extracting encoded data from radiation treatment sites in an EMR with great accuracy. This can be integrated into a repository of demographic, genomic, treatment, and outcome data to advance personalized oncologic care.

Received 06/26/2019 Review began $08 / 02 / 2019$ Review ended 10/25/2019 Published 10/28/2019

$\odot$ Copyright 2019 Walker et al. This is an open access article distributed under the terms of the Creative Commens $A$ th Creative Commons Attribution License CC-BY 3.0., which permits unrestricted use, distribution, and reproduction in any medium, provided the original author and source are credited.
Categories: Radiation Oncology

Keywords: radiation therapy, natural language processing, treatment site

\section{Introduction}

A National Radiation Oncology Registry (NROR) has been created through a collaboration between the Radiation Oncology Institute (ROI) and the American Society for Radiation Oncology (ASTRO) to develop a national database that will be used to study the clinical outcomes and patterns of care. Inherent in this effort is the need for automated tools to extract clinical information from radiation oncology electronic medical records (EMRs). Currently, radiation oncology-specific electronic medical records allow providers to input the treatment site using free text, leading to a glut of potential options. This paradigm creates great challenges in answering research questions that depend on assembling a cohort of patients who received similar treatments.

Previous studies have demonstrated the feasibility of extracting meaningful clinical data using natural language processing (NLP) tools from diagnoses [1], problem lists [2], pathology reports [3-4], and radiology reports [5-8]. These tools are not designed to handle the complexities of radiation therapy (RT) site names, which include many abbreviations specific to our field. The purpose of this study is to develop an NLP tool to extract encoded data from radiation treatment sites in an EMR.

\section{Technical Report}

For this analysis, information was obtained from the RT delivery (record and verify) electronic medical record (MOSAIQ ${ }^{\circledR}$, Elekta Care Management, Stockholm), which allows manual, free-text input of the desired treatment site. Treatment sites were extracted from all patients who completed treatment in our department from April 1, 2011, to April 30, 2013. A separate treatment site was entered for each radiation field. For example, a breast RT treatment might consist of three treatment sites in the RT prescription: 1) left breast field, 2) internal mammary chain field, and 3) supraclavicular field. In general, at that time, our department did not have a standardized nomenclature for labeling treatment sites. In addition, we practiced in a large department with multiple physicians per clinical service, leading to a large heterogeneity in the labeling of treatment sites. The study was deemed to be exempt from review by the Institutional Review Board as a quality improvement project.

A system was designed to extract the Unified Medical Language System (UMLS) concept codes using a sample of 11,018 unique site names from 31118 RT sites. Among those, 5500 unique site name strings that constituted approximately half of the sample were spared as a test set to evaluate the final system, and the remaining site name entries were used for system development.

As an initial requirement, we developed a dictionary and calculated n-gram statistics using UMLS concepts from related semantic types like Body Part, Organ, or Organ Component (T023), Body Location or Region (T029), Body Space or Junction (T030), or Spatial Concept (T082) that represented topographical entities within the body or other concepts. Although the majority of the concepts are covered by this UMLS subset, 


\section{Cureus}

there were still uncovered concepts specific to the radiation therapy domain. These concepts and common abbreviations from the development set were manually extracted to create a supplement terminology to overcome the shortcomings of UMLS.

The system first processed a site name into tokens and then each token was matched against the above dictionary. If the token was not in the dictionary, the English dictionary was used to identify the word. Because of arbitrary abbreviations ( for example, supraclavicular can be abbreviated as SCV, SCL, S/C, SC, S Clav, Sc V, Sclav, or SCLV), the system frequently returned an unknown token. In these cases, if there was no match, a list of possible candidates was obtained by preceding or following the token from the application terminology based on bigrams. For each candidate, the Levenshtein distance was calculated, and the closest word having the highest probability was chosen as the correct one. After this word identification step, the site name was processed to build terms, in comparison to the UMLS concept subset.

For the evaluation of the system, the application was run on the spared site names for test purposes. The output was compared to a review of site names by a radiation oncologist as the gold standard.

The mean number of times a unique treatment site was used among all patients was 2.82 (range 1450 ). There was an average of 2.2 sites per patient. Prior to extraction, the 20 most common unique treatment sites were used 4215 times (38.3\%) (Table 1).

\begin{tabular}{|c|c|c|c|c|c|}
\hline Site Name & Frequency & Concept Identified 1 & Concept Identified 2 & $\begin{array}{l}\text { Concept } \\
\text { Identified } 3\end{array}$ & $\begin{array}{l}\text { Conce } \\
\text { Identi1 }\end{array}$ \\
\hline Pelvis & 450 & $\begin{array}{l}\text { pelvis } \\
\text { [C0030797,C0559769,C0030786,C1279864:T023,T029,T030] }\end{array}$ & & & \\
\hline SCV & 428 & supraclavicular region [C0446461:T029] & & & \\
\hline Whole Brain & 298 & whole [C0444667:T081] & $\begin{array}{l}\text { brain } \\
\text { [C0006104,C1269537,C1882598,C1273723:T023,T029] }\end{array}$ & & \\
\hline whole brain & 266 & whole [C0444667:T081] & $\begin{array}{l}\text { Brain } \\
\text { [C0006104,C1269537,C1882598,C1273723:T023,T029] }\end{array}$ & & \\
\hline $\begin{array}{l}\text { Tumor Bed } \\
\text { Boost }\end{array}$ & 262 & tumor [C0027651,C3273930,C1578706:T191,T170,T033] & bed [C1547114:T082] & $\begin{array}{l}\text { Boost } \\
\text { [C1511253:T169] }\end{array}$ & \\
\hline Left Breast & 260 & Left Breast [C0222601:T023] & & & \\
\hline $\begin{array}{l}\text { Right Chest } \\
\text { Wall }\end{array}$ & 241 & right [C0205090:T082] & Chest wall [C0205076:T023] & & \\
\hline Right Breast & 239 & Right Breast [C0222600:T023] & & & \\
\hline $\begin{array}{l}\text { Left Chest } \\
\text { Wall }\end{array}$ & 206 & Left [C0205091:T082] & Chest wall [C0205076:T023] & & \\
\hline pelvis & 198 & $\begin{array}{l}\text { pelvis } \\
\text { [C0030797,C0559769,C0030786,C1279864:T023,T029,T030] }\end{array}$ & & & \\
\hline Boost & 194 & Boost [C1511253:T169] & & & \\
\hline Whole brain & 189 & whole [C0444667:T081] & $\begin{array}{l}\text { Brain } \\
\text { [C0006104,C1269537,C1882598,C1273723:T023,T029] }\end{array}$ & & \\
\hline $\begin{array}{l}\text { Prostate/Prox } \\
\text { sv }\end{array}$ & 159 & Prostate [C0033572,C1278980,C1882832:T023] & /[PUNC:T000] & $\begin{array}{l}\text { Proximal } \\
\text { [C0205107:T082] } \\
(222.0)\end{array}$ & $\begin{array}{l}\text { Semin } \\
\text { [C127! }\end{array}$ \\
\hline boost & 148 & Boost [C1511253:T169] & & & \\
\hline SCV-MLB & 147 & supraclavicular region [C0446461:T029] & midline block [:T169] & & \\
\hline SCV MLB & 139 & supraclavicular region [C0446461:T029] & midline block [:T169] & & \\
\hline Right breast & 134 & Right Breast [C0222600:T023] & & & \\
\hline Prostate & 129 & Prostate [C0033572,C1278980,C1882832:T023] & & & \\
\hline WBRI & 128 & whole-brain radiotherapy [C1520143:1061] & & & \\
\hline
\end{tabular}

TABLE 1: The 20 most frequent treatment sites with natural language processing extracted clinical concepts

SCV: supraclavicular

The customized NLP solution displayed great gains as compared to other systems, with a recall of 0.99 and a precision of 0.99 (Table 2). 


\section{Cureus}

\begin{tabular}{|l|l|l|}
\hline Application & Recall TP/(TP+FN) & Precision TP/(TP+FP) \\
\hline MetaMap & 0.51 & 0.75 \\
CTAKES & 0.46 & 0.85 \\
Customized NLP System & 0.99 & 0.99 \\
\hline
\end{tabular}

TABLE 2: Recall and precision for the customized natural language processing system, as compared to two common NLP tools (MetaMap and cTAKES)

NLP: natural language processing; cTAKES: clinical text analysis and knowledge extraction system

The most common treatment site was whole brain RT, which was entered using 27 distinct terms for a total of 1063 times (Table 3).

\begin{tabular}{|c|c|}
\hline Name & Frequency \\
\hline Whole Brain & 298 \\
\hline whole brain & 266 \\
\hline Whole brain & 189 \\
\hline WBRT & 128 \\
\hline Brain & 113 \\
\hline brain & 21 \\
\hline wbxrt & 8 \\
\hline WHOLE BRAIN & 6 \\
\hline Whole brain 2 & 3 \\
\hline whole brain PCI & 3 \\
\hline whole Brain & 3 \\
\hline Whole Brain \#2 & 3 \\
\hline Whole Brain PCI & 3 \\
\hline Whole Brain Retreat & 2 \\
\hline WHole Brain & 2 \\
\hline whole brain 1 & 2 \\
\hline Whole Brain RT & 2 \\
\hline WBRT (PCI) & 2 \\
\hline whole brain-2 & 1 \\
\hline Whole Brain photons & 1 \\
\hline $\mathrm{PCl}$, whole brain & 1 \\
\hline Whole brain/eyes/BOS & 1 \\
\hline Whole Brain Proton & 1 \\
\hline whole brain modulate & 1 \\
\hline whole brain 2 & 1 \\
\hline Whole brain Primary & 1 \\
\hline Whole Brain-PCI & 1 \\
\hline
\end{tabular}

TABLE 3: Variations in describing treatment site for whole-brain radiation therapy

PCl: percutaneous coronary intervention; WBRT: whole-brain radiation therapy; WBXRT: whole-brain radiotherapy

\section{Discussion}


This study analyzed a cohort of RT treatment sites and developed a customized NLP system that can extract structured data with very high recall and precision compared to non-customized tools. A method to extract structured data from RT treatment sites has not been described in the literature.

This tool can be incorporated into an institutional data warehouse as a repository of integrated genomic sequencing data, treatment details, and outcome data. This data will allow us to make better treatment decisions and predict individual patients' risk of acute and long-term toxicity due to oncologic therapy and thus further personalize their care.

Currently, the majority of cancer registries and departmental databases rely on manual coding to determine receipt of RT. With the widespread adoption of EMRs, automated coding will become increasingly important. NLP has the potential to facilitate this reporting in a structured, meaningful way. This tool could automate the reporting of treatment fields to improve the quality and accuracy of retrospective and prospective research, thus improving their meaningful use. In addition, similar tools can be developed for other radiation oncology applications such as extracting coded data from dose-volume histograms (DVHs).

This study has certain limitations, which need to be addressed. While these results are compelling, they only apply to our unique clinical workflow. Some institutions may have a more structured process for entering treatment sites, leading to differing results with a similar tool.

\section{Conclusions}

In summary, we developed an NLP tool to extract encoded data from radiation treatment sites in an EMR. This can be integrated into a repository of demographic, genomic, treatment, and outcome data to advance personalized oncologic care.

\section{Additional Information \\ Disclosures}

Human subjects: Consent was obtained by all participants in this study. MD Anderson Cancer Center issued approval NA. The study was deemed to be exempt from review by the Institutional Review Board as a quality improvement project. Animal subjects: All authors have confirmed that this study did not involve animal subjects or tissue. Conflicts of interest: In compliance with the ICMJE uniform disclosure form, all authors declare the following: Payment/services info: All authors have declared that no financial support was received from any organization for the submitted work. Financial relationships: Gary Walker declare(s) a grant from Keck Center for Interdisciplinary Bioscience Training of the Gulf Coast Consortia. Dr. Walker was supported by a training fellowship from the Keck Center for Interdisciplinary Bioscience Training of the Gulf Coast Consortia (Grant No. T15 LM007093). Other relationships: All authors have declared that there are no other relationships or activities that could appear to have influenced the submitted work.

\section{References}

1. Zeng QT, Goryachev S, Weiss S, Sordo M, Murphy SN, Lazarus R: Extracting principal diagnosis, comorbidity and smoking status for asthma research: evaluation of a natural language processing system. BMC Med Inform Decis Mak. 2006, 6:30. 10.1186/1472-6947-6-30

2. Meystre S, Haug PJ: Automation of a problem list using natural language processing . BMC Med Inform Decis Mak. 2005, 5:30. 10.1186/1472-6947-5-30

3. Xu H, Friedman C: Facilitating research in pathology using natural language processing . AMIA Annu Symp Proc. 2003, 2003:1057.

4. Imler TD, Morea J, Kahi C, Imperiale TF: Natural language processing accurately categorizes findings from colonoscopy and pathology reports. Clin Gastroenterol Hepatol. 2013, 11:689-694. 10.1016/j.cgh.2012.11.035

5. Soysal E, Cicekli I, Baykal N: Design and evaluation of an ontology based information extraction system for radiological reports. Comput Biol Med. 2010, 40:900-911. 10.1016/j.compbiomed.2010.10.002

6. Lacson R, Khorasani R: Practical examples of natural language processing in radiology . J Am Coll Radiol. 2011, 8:872-874. 10.1016/j.jacr.2011.09.010

7. Friedman C, Alderson PO, Austin JH, Cimino JJ, Johnson SB: A general natural-language text processor for clinical radiology. J Am Med Inform Assoc. 1994, 1:161-174. 10.1136/jamia.1994.95236146

8. Hripcsak G, Austin JH, Alderson PO, Friedman C: Use of natural language processing to translate clinical information from a database of 889,921 chest radiographic reports. Radiology. 2002, 224:157-163. 10.1148/radiol.2241011118 